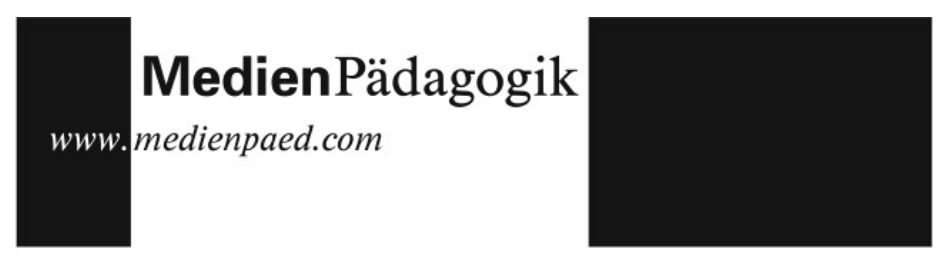

Rezensionen

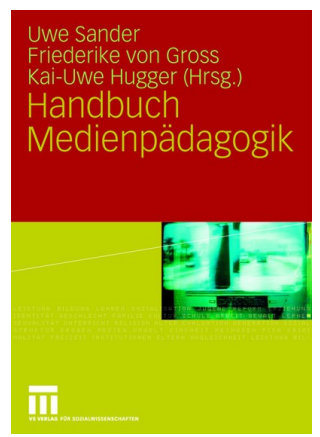

Uwe Sander, Friederike von Gross, Kai-Uwe Hugger (Hrsg.)

Handbuch Medienpädagogik

Wiesbaden: VS Verlag, 2008. 602 Seiten

ISBN: 13 978-3531150161

$€$ 49.90; CHF 85.-

\title{
Grundlagen einer facettenreichen Disziplin
}

Inzwischen liegen der Medienpädagogik einige Nachschlagewerke vor, die das Fach in seiner Vielschichtigkeit fassbar zu machen suchen. Das von Uwe Sander, Friederike von Gross und Kai-Uwe Hugger herausgegebene «Handbuch Medienpädagogik» hebt sich vor allem durch die Breite der behandelten Themen, die interdisziplinäre Ausrichtung und die Vielzahl der hier versammelten Autor/innen ab. In sieben Kapiteln «will [es] sowohl Studierenden, pädagogischen Berufspraktiker als auch Wissenschaftlern einen fundierten und systematisch aufgebauten Überblick über Theorie, Forschung, Geschichte, gegenwärtige Diskussionspunkte und Handlungsfeld der noch verhältnismäßig jungen erziehungswissenschaftlichen Teildisziplin liefern.» (Vorwort, 13)

Das erste Kapitel des 600 Seiten starken Werks behandelt "Geschichte und Strömungen der Medienpädagogik». In der Zusammenschau weisen die Artikel die zentralen Perspektiven und Zielhorizonte auf, die bis heute die Disziplin beeinflussen und prägen: von Reformpädagogik, über Bewahrpädagogik und bildungstechnologischer bis hin zu kritisch-emanzipativer und handlungsorientierter Medienpädagogik. Im umfangreichen zweiten Kapitel skizzieren die Autor/innen theoretische Grundlagen des Faches. In vier Unterkapiteln unterscheiden sie dabei «erziehungswissenschaftliche Theorien» (Stichworte sind hier u.a. Mediensozialisation, Medienkompetenz, Medienbildung, Mediendidaktik), "sozialwissenschaftliche und psychologische Theorien» (wie z.B. Cultural Studies, Konstruktivismus, Systemtheorie), «medienphilosophische Theorien» (entlang von Denkern wie Benjamin, Cassirer, 
Derrida, Flusser) sowie «Theorieansätze und Hypothesen» (z.B. Wissenskluft-Perspektive oder Agenda-Setting). Die Darstellung der einzelnen Theorien und Ansätze geschieht prägnant auf je ca. fünf bis acht Seiten. Im Anschluss an Geschichte und Theorie widmet sich das dritte Kapitel methodologischen und methodischen Grundlagen medienpädagogischer Forschung. Zwei einführende Artikel zur qualitativen und quantitativen Medienforschung eröffnen das Panorama, innen folgen Darstellungen unterschiedlicher methodischer Zugänge, deren Spektrum u.a. Rezeptions-, Biografieund Medienwirkungsforschung umfasst. Das vierte Kapitel des Buches ist mit «Medienentwicklung und Medienpädagogik» überschrieben. Berücksichtigt sind hierbei Buch, Zeitungen und Zeitschriften, Kino, Radio, Fernsehen und Video sowie Neue Medien. Unklar bleibt, aus welchem Grund Medien wie Mobiltelefon und Computerspiel oder auch übergreifende Phänomene, z.B. Medienkonvergenz, keine eingehende Thematisierung gefunden haben. Im fünften Kapitel werden «Diskussionsfelder der Medienpädagogik» vorgestellt. Elf Artikel bieten eine Auseinandersetzung mit Problemfeldern wie Globalisierung, Identitätskonstruktionen, Jugendkulturen, Migration oder Lehren und Lernen in digitalen Welten. Das sechste Kapitel schlägt schließlich gänzlich die Brücke zur Praxis und beleuchtet Ansätze in pädagogischen Aufgaben- und Handlungsfeldern wie Kindergarten und Schule, der außerschulischen Medienarbeit und der Bürgermedien. Ein eigenes Unterkapitel mit insgesamt drei Abschnitten ist dem Kinder- und Jugendmedienschutz gewidmet. Vorgestellt werden seine Geschichte, die beteiligten Institutionen sowie die gesetzlichen Grundlagen. Es sei darauf hingewiesen, dass auch die praxisorientierten Kapitel der theoretisch-reflektierenden Perspektive des Handbuches treu bleiben. Im Mittelpunkt steht die Darstellung grundsätzlicher medienpädagogischer bzw. -didaktischer Herangehensweisen und Prinzipien. Konkrete Praxismodelle oder Hinweise für die Planung und Durchführung von Projekten gibt es nur am Rande. Das siebente Kapitel des Handbuches thematisiert beruflich-professionelle Aspekte der Medienpädagogik und zeigt Berufswege und Tätigkeitsfelder auf.

Betrachtet man das Buch als Ganzes, so bietet dieses eine Übersicht über die vielschichtigen und vielfältigen Facetten eines kaum klar zu umreißenden Fachbereiches. Das «Handbuch Medienpädagogik» und mit ihm die 


\section{Rezensionen}

zahlreichen Expert/innen geben einen umfassenden Einblick in Historie, Bezugstheorien, Methoden und Diskussionsfelder der Disziplin. Diejenigen, die bereits «eingelesen» sind, können hier nachschlagen, Wissen auffrischen und vertiefen, stoßen bestenfalls auf weiterführende Themen und Fragestellungen oder finden gänzlich neue Anregungen. In der Breite der eingebrachten Diskurse aus unterschiedlichen wissenschaftlichen Disziplinen wie Pädagogik, Soziologie oder Kommunikations- und Medienwissenschaft entfaltet der Band seine Stärke.

Ein Problem ist allerdings darin zu sehen, dass die Zusammenstellung und Zusammenführung allein noch keine Orientierung bringt. Gerade für den Einstieg in die Medienpädagogik/Medienbildung sind Einordnung und Verortung aber von zentraler Bedeutung - und von einem «Handbuch» sind diese zu erwarten. Im Buch finden sich lediglich zwei ordnende Prinzipien: die Gliederung, d.h. das Inhaltsverzeichnis, sowie das Stichwortverzeichnis. Darüber hinaus wären jedoch einleitende Überblicksartikel, die die Leser/innen etwa auf der Ebene der sieben Großkapitel auf das Kommende vorbereiten, ebenso hilfreich wie zusätzliche Querverweise zwischen den Einzelbeiträgen. Auch fällt auf, dass die Diskussion derjenigen Referenztheorien und -ansätze, die die Medienpädagogik als quer liegende Disziplin zwar prägen, aber keinen genuin medienpädagogischen Bezug haben, ganz unterschiedlich ausfällt. Während sich einige Autor/innen um eine Übertragung auf das Feld der Medienpädagogik bemühen, belassen es andere dabei, die Grundzüge der jeweiligen Theorien, Denker oder Ansätze vorzustellen. Insbesondere in der Darstellung der sozialwissenschaftlichen und psychologischen Theorien, der medienphilosophischen Theorien und der Theorieansätze und Hypothesen unterscheidet sich das Handbuch deshalb mitunter nur wenig von anderen Einführungswerken, etwa in die Kommunikations-, Publizistik- und/oder Medienwissenschaft bzw. in die Medientheorie. Das muss kein Manko sein, wenn man sich vor Augen hält, dass die Grenzen der Disziplin(en) ohnehin fließend sind, sollte beim Lesen und auch einer etwaigen Kaufentscheidung jedoch mitbedacht sein.

Mandy Schiefner, Wolfgang Reißmann 